\title{
Enabling Interactive Transcription in an Indigenous Community
}

\author{
Éric Le Ferrand, ${ }^{1,2}$ Steven Bird, ${ }^{1}$ and Laurent Besacier ${ }^{2}$ \\ ${ }^{1}$ Northern Institute, Charles Darwin University, Australia \\ ${ }^{2}$ Laboratoire Informatique de Grenoble, Université Grenobles Alpes, France
}

\begin{abstract}
We propose a novel transcription workflow which combines spoken term detection and humanin-the-loop, together with a pilot experiment. This work is grounded in an almost zero-resource scenario where only a few terms have so far been identified, involving two endangered languages. We show that in the early stages of transcription, when the available data is insufficient to train a robust ASR system, it is possible to take advantage of the transcription of a small number of isolated words in order to bootstrap the transcription of a speech collection.
\end{abstract}

\section{Introduction}

In remote Aboriginal communities in Australia, many efforts are made to document traditional knowledge including rock art, medicinal plants, and food practices. While it may be relatively straightforward to capture spoken content, transcription is time-consuming and has been described as a bottleneck (Brinckmann, 2009). Transcribing is often seen as an obligatory step, to facilitate access to audio. Efforts have been made to speed up this process using speech recognition systems, but the amount of data available in Indigenous language contexts is usually too limited for such methods to be effective.

Recent research has shown the efficacy of spoken term detection methods when data are scarce (Menon et al., 2018a; Menon et al., 2018b). Taking advantage of the transcription of a few words would allow us to propagate it through the speech collection and thus assist language workers in their regular transcription work. So-called "sparse transcription" would be also a way to navigate a speech collection and allow us to be selective about what needs to be transcribed (Bird, 2020). Several tools exist for manual transcription, such as Elan and Praat (Wittenburg et al., 2006; Boersma and Weenink, 1996). However such transcriptions are often made in isolation from the speech community (First Languages Australia, 2014), and so we miss out on the opportunity to take advantage of the interests and skills of local people to shape and carry out the transcription work.

We present a fieldwork pipeline which combines automatic speech processing and human expertise to support speech transcription in almost-zero resource settings. After giving the background, we detail the workflow and propose a pilot experiment on two very low-resource corpora.

\section{Background}

Existing approaches to automatic transcription of endangered languages involve methods that have been developed for automatic speech recognition. While a few hours of transcribed speech can be enough to train single-speaker models (Gupta and Boulianne, 2020b), speaker-independent models require a large amount of training data to produce useful transcriptions (Gupta and Boulianne, 2020a; Foley et al., 2018). Moreover, they draw language workers and speakers into the time-consuming task of exhaustive transcription, forcing them to transcribe densely, including passages that may be difficult or impossible given the early state of our knowledge of the language. A more suitable approach, we believe, involves beginning with stretches of speech where we have the greatest confidence, and only later tackling the more difficult parts.

This work is licensed under a Creative Commons Attribution 4.0 International Licence. Licence details: http:// creativecommons.org/licenses/by/4.0/. 
Spoken term detection involves retrieving a segment in a speech collection, given an example. With this method, it is possible to sparsely transcribe the corpus, i.e., take advantage of an existing transcription (or a list of spoken words) and identify tokens throughout the collection (Bird, 2020).

Dynamic Time Warping (DTW) (Sakoe and Chiba, 1978) and its more advanced versions (Park and Glass, 2005; Jansen and Van Durme, 2011) can be used to match a query in a speech collection, but they can be computationally expensive. DTW "aligns two sequences of feature vectors by warping their time axes to achieve an optimal match" (Menon et al., 2018a). A common method is to compute DTW, sliding the spoken query over each utterance in the corpus. In addition, several speech representations have been considered in the literature for the task of word-spotting. Kamper et al. (2015) and Menon et al. (2019) explore feature extraction from autoencoders (AE) and correspondence autoencoders (cAE) and show how top-down constraints can improve the quality of the hidden representation for spoken term detection tasks. Schneider et al. (2019) introduce wav2vec, a self-supervised model for speech representation learning which is based on contrastive predictive coding, and apply this to supervised ASR.

\section{Proposed Workflow}

\subsection{Interactive and Sparse Transcription}

The key idea of sparse transcription is to use spoken term detection methods to sparsely transcribe a speech collection, beginning with a small collection of spoken terms. A spoken term is defined as a chunk of speech considered meaningful by a speaker, which may be a morph, a word, or a multiword expression. This collection of terms can be a list of keywords (or morphs) recorded in isolation by a speaker. Equally, it can be obtained by extracting audio clips from a speech collection.

We set up the workflow as shown in Figure 1. We begin with a lexicon of size $s$ and a speech collection (Fig. 1a). This lexicon is composed of speech terms and their orthographic transcriptions $w_{1} \ldots w_{n}$. We use a spoken term detection method to retrieve speech terms in the collection that match those in the lexicon (Fig. 1b). The system presents the $n$ most confidently identified terms and presents them for verification (Fig. 1c). False positives are corrected, i.e., erased from the transcription (Fig. 1d). We clip out from the utterances the speech terms correctly retrieved and add them to the lexicon as extra samples of a given entry (Fig. 1e). We allow a single entry to have maximum of $m$ extra examples. We manually collect new speech units with a speaker, add them to the lexicon (Fig. 1f) and start a new iteration (Fig. 1b). We apply this for $i$ iterations with a lexicon growing each time. The size $s$ of the lexicon, $n$ number of words checked, maximum $m$ of extra examples for each word, and number $i$ of iterations are hyperparameters which vary according to the contingencies of the fieldwork.

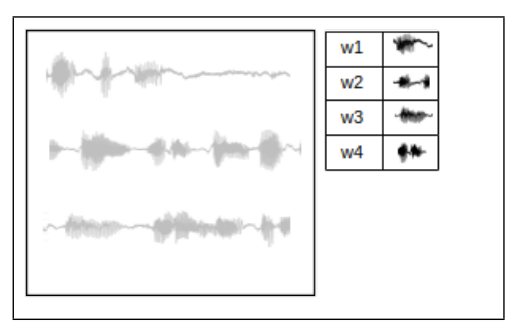

(a) Starting point

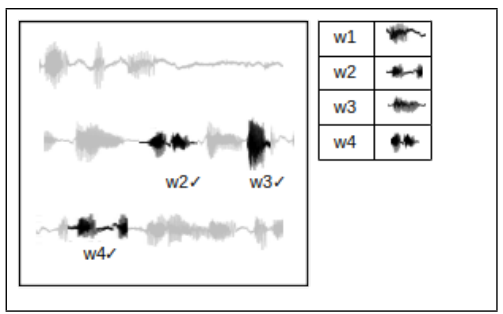

(d) Confirmed hits

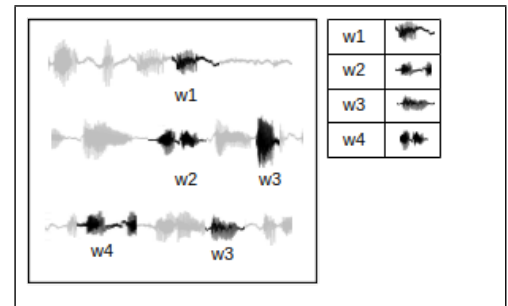

(b) Spoken term detection

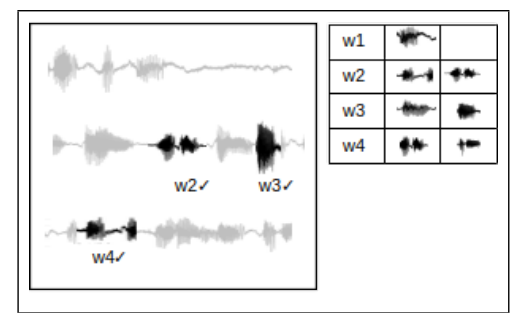

(e) Extra examples added to lexicon

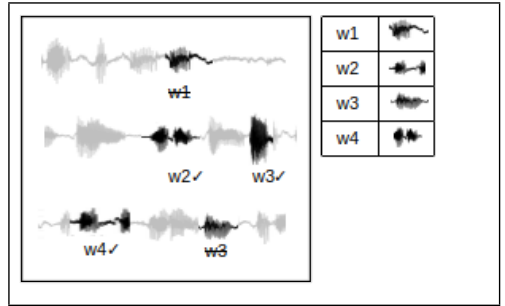

(c) Manual confirmation

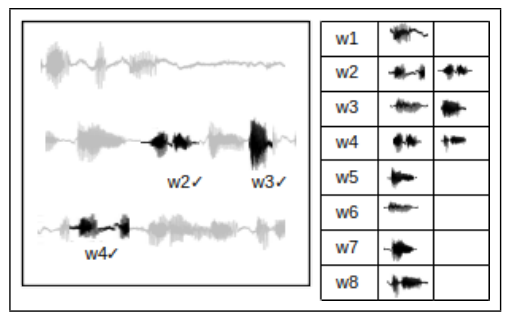

(f) Expanded lexicon

Figure 1: One iteration of our Interactive Sparse Transcription Workflow 


\section{Pilot experiment: simulating this interactive scenario}

Speech data. We apply the pipeline to two corpora. The first one is a $4 \mathrm{~h} 30 \mathrm{~m}$ corpus in Mboshi ${ }^{1}$ (Godard et al., 2018), a Bantu language spoken in Congo Brazaville (ISO mdw). It consists of 5,130 utterances sentence and word-aligned with an orthographic transcription. The utterances are elicited from text. This corpus contains only three speakers, of which one is responsible for $70 \%$ of the corpus. The second corpus is a very small $(0 \mathrm{~h} 20 \mathrm{~m})$ corpus in Kunwinjku, an Australian Aboriginal language (ISO gup). It consists of 301 utterances aligned with an orthographic transcription. A forced alignment at the wordlevel has been created using the MAUS aligner (Kisler et al., 2017). The corpus contains 4 guided tours of the same town and one guided tour of another Aboriginal site. Each tour has been produced by a different speaker. To create initial and expanded lexicons, we select the 100 and 60 most frequent words bigger than 3 syllables for Mboshi and Kunwinjku respectively, in order to avoid words which are too short. A speech occurrence of each entry is extracted from the speech collection using the word-level alignments.

Acoustic features. In this interactive process, we explore several speech representations to identify those that are most suited to the pipeline, namely mel-frequency cepstral coefficients (MFCC) and perceptual linear prediction (PLP) features. We also use the hidden representation of an auto-encoder (AE) and correspondence auto-encoder (cAE). For this we use architecture of (Kamper et al., 2015) to self-train a 5-layer stacked AE (instead of 8) on $4 \mathrm{~h}$ of Mboshi and 2h of Kunwinjku (YouTube videos). The $\mathrm{cAE}$ is trained using similar segments extracted from the speech collections with an unsupervised term discovery tool (Jansen and Van Durme, 2011). Finally we also use wav2vec representations (Schneider et al., 2019). The wav2vec model is either trained from scratch on Mboshi and Kunwinjku $\left(\mathrm{w} 2 \mathrm{v} \_\mathrm{mb}\right.$ and $\mathrm{w} 2 \mathrm{v} \_$kun) or it is adapted from an original English model ( $\mathrm{w} 2 \mathrm{v} \_\mathrm{en} \_\mathrm{mb}$ and $\mathrm{w} 2 \mathrm{v} \_$en $k \mathrm{kun}$ ). We experiment with these features, with and without mean and variance normalisation (MVN) (Strand and Egeberg, 2004).

Sparse transcription experiments. The workflow described in section 3.1 is applied for 5 iterations for Mboshi and 3 iterations for Kunwinjku with an initial lexicon consisting of 20 words. 20 new words are added at the lexicon at each iteration. We end up with a 100 word lexicon for Mboshi and 60 words for Kunwinjku. The 10 best hits per word are checked $(n=10)$ and a maximum of 5 extra examples per word $(m=5)$ are added to the lexicon for both corpora. In addition, in order to avoid unnecessary verification in case the DTW score is too low, the worst score of the correct words checked during the first iteration is used as a threshold for the following iteration. To simulate human verification (Fig. 1c) we directly compare the hits output by the system with the gold transcriptions of the corpora.

\section{Results: impact of speech feature representations in the workflow}

We report the results of this new workflow in Table 1. The average precision scores (AP) correspond to the mean of the precision of the workflow computed at each iteration. The final recall is defined as the number of items retrieved from the full corpus $X$ out of the number of all retrievable items in the corpus, i.e., the intersection of the lexicon $L$ and the corpus $C, X /(L \cap C)$. In other words, this recall corresponds to the coverage of the lexicon related to its tokens in the speech collection. The impact of MVN is detailed only for the basic representations (PLP and MFCCs) since normalisation did not show any major influence on the neural representations (AE, cAE and $w 2 v$ ). We can make the following comments from the results shown in Table 1: (a) mean and variance normalisation (MVN) is important to improve results of basic (PLP and MFCC) features. Figure 2 shows that normalisation improves retrieval when the query term and the search term are pronounced by different speakers; (b) neural AE and cAE features are normalized-by-design but do not lead necessarily to better performance than PLP and MFCC; and (c) representations provided by wav $2 \mathrm{vec}$ are not efficient. The small size of the corpora might be the main obstacle when training efficient self-supervised models for learning speech representations.

Speaker diversity in the two corpora (Sec. 4) has an impact on the final results. For Mboshi $70 \%$ of the words to be retrieved in the speech collection are pronounced by the same speaker in the final

\footnotetext{
${ }^{1}$ https://github.com/besacier/mboshi-french-parallel-corpus
} 


\begin{tabular}{l|l|l|l}
\hline Features & MVN & AP & final recall \\
\hline mfcc & no & 23.87 & 16.78 \\
mfcc & yes & $\mathbf{3 2 . 6 7}$ & $\mathbf{2 3 . 3 7}$ \\
plp & no & 23.89 & 16.86 \\
plp & yes & 31.64 & 23.03 \\
w2v_mb & no & 24.57 & 16.88 \\
w2v_en_mb & no & 19.23 & 13.72 \\
AE & no & 31.93 & 21.51 \\
cAE & no & 27.31 & 20.70 \\
\hline
\end{tabular}

\begin{tabular}{l|l|l|l}
\hline Features & MVN & AP & final recall \\
\hline mfcc & no & 15.42 & 30.82 \\
mfcc & yes & 20.82 & 42.84 \\
plp & no & 15.21 & 32.67 \\
plp & yes & $\mathbf{2 2 . 5 5}$ & 44.89 \\
w2v_kun & no & 5.39 & 15.72 \\
w2v_en_kun & no & 5.45 & 15.87 \\
AE & no & 22.07 & $\mathbf{4 5 . 3 0}$ \\
cAE & no & 21.88 & 40.37 \\
\hline
\end{tabular}

Table 1: Results for the Mboshi corpus (left) and for the Kunwinjku corpus (right)

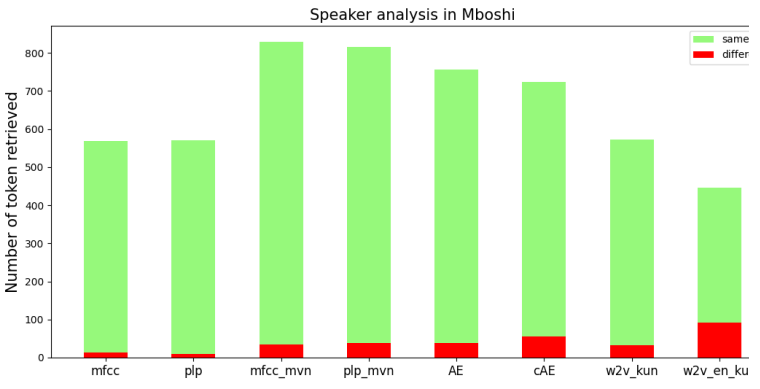

(a)

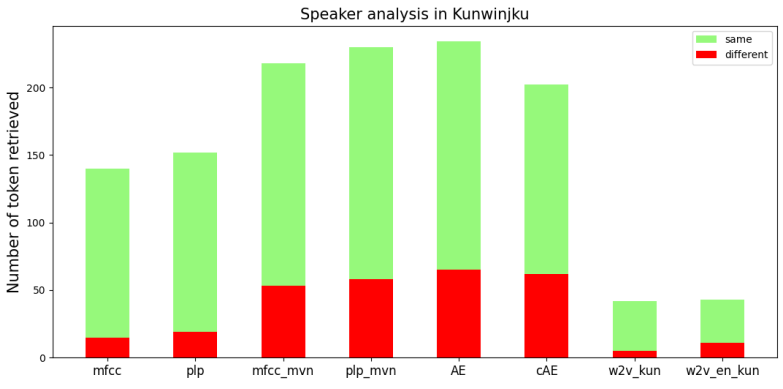

(b)

Figure 2: Proportion of same-speaker/different-speaker retrieval for each representation

lexicon and $37 \%$ for Kunwinjku. Figure 2 reports the proportion of tokens retrieved between same speakers and different speakers over the total number of tokens retrieved for Mboshi and Kunwinjku. The first observation is that the pipeline mostly retrieves terms pronounced by the speaker. It is clear that MVN improves performance for both same-speaker and different-speaker retrieval. We also note that, while wav2 vec representation is overall less performant, it seems promising for extracting more speaker-independent representations, as illustrated by the results obtained with w2v_en_mb features. Future work will investigate this by leveraging more raw speech in Mboshi and Kunwinjku for training better self-supervised (wav2vec) models.

Regarding false positives (Table 2), the first errors we can observe are different spellings of words (in the gold transcription) referring to the same meaning (e.g., namekke / nemekke "that one"). Since Kunwinjku is primarily a spoken language, variable spellings are common. Moreover, because of the morphological complexity of the language, many of the top false positives are actually inflectional variants of the query term (e.g., balanda / balanda-ken "white man / from the white man"). Allowing matching at a smaller granularity could be a way to achieve wider coverage of the speech collection.

\begin{tabular}{l|l|l|l}
\hline Query & False Positive & query translation & hit translation \\
\hline nahne & mahne & it (pronoun) & this (demonstrative) \\
nemekke & namekke & that one & that one (other spelling) \\
balanda & balanda-ken & white man & of the white man (genitive) \\
bininj & bininj-beh & man & from the man \\
nemekke & yekke & that one & dry season \\
mahni & mahne & this (demonstrative) & this (other spelling) \\
\hline
\end{tabular}

Table 2: Top false positive generated by the spoken term detection system in Kunwinjku 


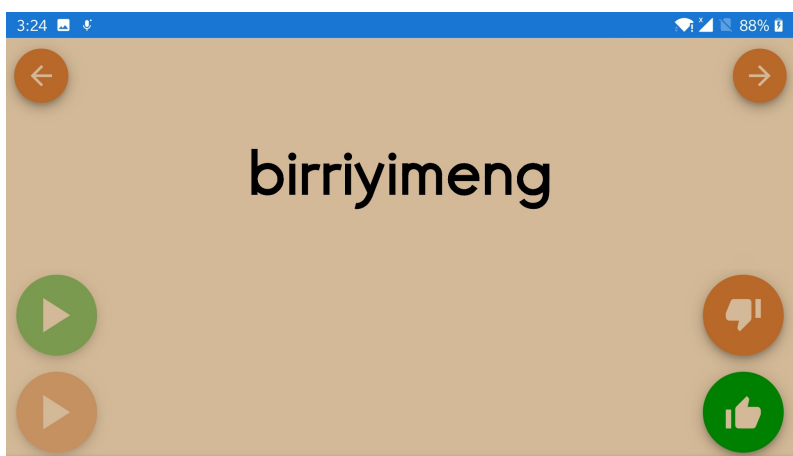

(a) screenshot of the app

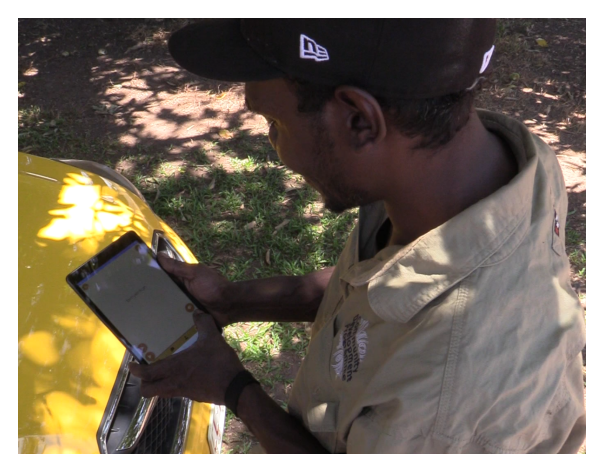

(b) deployment of the app

Figure 3: Lexical confirmation app

\section{Deployment}

Building on prior work developing mobile tools for language documentation (Bettinson and Bird, 2017), we have begun to explore methods for deploying the pipeline in a remote community. While the first step of identification of new words is straightforward, the task of lexical confirmation might be much more complex to apply. The members of an Aboriginal community might not be familiar with technologies and they are not necessarily literate (in the narrow western sense). Taking into account these constraints, we built a lexical confirmation app and trialled it on a small lexicon (Fig. 3).

The idea would be to load the output of the spoken term detection system into the app. Then a speaker can listen to the query, listen to the utterance where we expect the query to be found, and confirm if the utterance contains the query, (Fig.1c)

\section{Conclusion}

We investigated the use of spoken term detection methods as an alternative to the usual methods that have been inspired by automatic speech recognition, and which require exhaustive transcription even for passages which exceed the present state of our knowledge about the language. Instead we devised a workflow based on spoken term detection, and simulated it for two small corpora: one in Mboshi, one in Kunwinjku. The simulations of this workflow show that, with well chosen speech representations, we may have a viable approach for rapidly bootstrapping transcriptions of large collections of speech in endangered languages. The next step of this work would be to design methods to involve Indigenous people in tasks such as the construction of the lexicon or the confirmation of the output of our system for a deployment of this workflow in a remote community.

\section{Acknowledgements}

We are grateful to the Bininj people of Northern Australia for the opportunity to work in their community, and particularly to artists at Injalak Arts and Craft (Gunbalanya) and to the Warddeken Rangers (Kabulwarnamyo). Our thanks to several anonymous reviewers for helpful feedback on earlier versions of this paper. The lexical confirmation app presented in this paper has been designed by Mat Bettinson, at Charles Darwin University. This research was covered by a research permit from the Northern Land Council, ethics approved from CDU and was supported by the Australian government through a PhD scholarship, and grants from the Australian Research Council and the Indigenous Language and Arts Program. 


\section{References}

Mat Bettinson and Steven Bird. 2017. Developing a suite of mobile applications for collaborative language documentation. In Proceedings of the 2nd Workshop on the Use of Computational Methods in the Study of Endangered Languages, pages 156-164.

Steven Bird. 2020. Sparse transcription. Computational Linguistics, 46(4).

Paul Boersma and David Weenink. 1996. Praat, a system for doing phonetics by computer, version 3.4. Institute of Phonetic Sciences of the University of Amsterdam, Report, 132:182.

Caren Brinckmann. 2009. Transcription bottleneck of speech corpus exploitation. Proceedings of the 2nd Colloquium on Lesser Used Languages and Computer Linguistics, pages 165 - 179.

First Languages Australia. 2014. Angkety Map: Digital resource report. Technical report. https://www . firstlanguages.org.au/images/fla-angkety-map.pdf; accessed Nov 2020.

Ben Foley, Joshua T Arnold, Rolando Coto-Solano, Gautier Durantin, T Mark Ellison, Daan van Esch, Scott Heath, Frantisek Kratochvil, Zara Maxwell-Smith, David Nash, et al. 2018. Building speech recognition systems for language documentation: The CoEDL Endangered Language Pipeline and Inference System (ELPIS). In Proceedings of The 6th International Workshop on Spoken Language Technologies for Under-Resourced Languages, pages 205-209.

Pierre Godard, Gilles Adda, Martine Adda-Decker, Juan Benjumea, Laurent Besacier, Jamison Cooper-Leavitt, Guy-Noel Kouarata, Lori Lamel, Hélène Bonneau-Maynard, Markus Mueller, et al. 2018. A very low resource language speech corpus for computational language documentation experiments. In Proceedings of the 11th International Conference on Language Resources and Evaluation, pages 3366-70.

Vishwa Gupta and Gilles Boulianne. 2020a. Automatic transcription challenges for Inuktitut, a low-resource polysynthetic language. In Proceedings of the 12th Language Resources and Evaluation Conference, pages $2521-27$.

Vishwa Gupta and Gilles Boulianne. 2020b. Speech transcription challenges for resource constrained indigenous language Cree. In Proceedings of the 1st Joint Workshop on Spoken Language Technologies for UnderResourced Languages and Collaboration and Computing for Under-Resourced Languages (CCURL), pages 362-367.

Aren Jansen and Benjamin Van Durme. 2011. Efficient spoken term discovery using randomized algorithms. In 2011 IEEE Workshop on Automatic Speech Recognition \& Understanding, pages 401-406. IEEE.

Herman Kamper, Micha Elsner, Aren Jansen, and Sharon Goldwater. 2015. Unsupervised neural network based feature extraction using weak top-down constraints. In 2015 IEEE International Conference on Acoustics, Speech and Signal Processing (ICASSP), pages 5818-22. IEEE.

Thomas Kisler, Uwe Reichel, and Florian Schiel. 2017. Multilingual processing of speech via web services. Computer Speech and Language, 45:326-347.

Raghav Menon, Herman Kamper, John Quinn, and Thomas Niesler. 2018a. Fast ASR-free and almost zeroresource keyword spotting using DTW and CNNs for humanitarian monitoring. In Proceedings of Interspeech 2018, pages 2608-12.

Raghav Menon, Herman Kamper, Emre Yilmaz, John Quinn, and Thomas Niesler. 2018b. ASR-Free CNN-DTW keyword spotting using multilingual bottleneck features for almost zero-resource languages. In Proceedings of the 6th International Workshop on Spoken Language Technologies for Under-Resourced Languages, pages $182-186$

Raghav Menon, Herman Kamper, Ewald van der Westhuizen, John Quinn, and Thomas Niesler. 2019. Feature exploration for almost zero-resource ASR-free keyword spotting using a multilingual bottleneck extractor and correspondence autoencoders. Proceedings of Interspeech 2019, pages 3475-3479.

Alex Park and James R Glass. 2005. Towards unsupervised pattern discovery in speech. In IEEE Workshop on Automatic Speech Recognition and Understanding, pages 53-58. IEEE.

Hiroaki Sakoe and Seibi Chiba. 1978. Dynamic programming algorithm optimization for spoken word recognition. IEEE Transactions on Acoustics, Speech, and Signal Processing, 26:43-49.

Steffen Schneider, Alexei Baevski, Ronan Collobert, and Michael Auli. 2019. Wav2vec: Unsupervised pretraining for speech recognition. Proceedings of Interspeech 2019, pages 3465-69. 
Ole Morten Strand and Andreas Egeberg. 2004. Cepstral mean and variance normalization in the model domain. In ISCA Tutorial and Research Workshop on Robustness Issues in Conversational Interaction.

Peter Wittenburg, Hennie Brugman, Albert Russel, Alex Klassmann, and Han Sloetjes. 2006. Elan: a professional framework for multimodality research. In 5th International Conference on Language Resources and Evaluation, pages $1556-15$. 